\title{
ARTICLE
}

\section{Efficient near-infrared up-conversion photoluminescence in carbon nanotubes}

\author{
Naoto Akizuki ${ }^{1}$, Shun Aota ${ }^{1}$, Shinichiro Mouri ${ }^{1}$, Kazunari Matsuda ${ }^{1}$ \& Yuhei Miyauchi, ${ }^{1,2,3}$
}

Photoluminescence phenomena normally obey Stokes' law of luminescence according to which the emitted photon energy is typically lower than its excitation counterparts. Here we show that carbon nanotubes break this rule under one-photon excitation conditions. We found that the carbon nanotubes exhibit efficient near-infrared photoluminescence upon photoexcitation even at an energy lying $>100-200$ meV below that of the emission at room temperature. This apparently anomalous phenomenon is attributed to efficient one-phononassisted up-conversion processes resulting from unique excited-state dynamics emerging in an individual carbon nanotube with accidentally or intentionally embedded localized states. These findings may open new doors for energy harvesting, optoelectronics and deep-tissue photoluminescence imaging in the near-infrared optical range.

\footnotetext{
${ }^{1}$ Institute of Advanced Energy, Kyoto University, Uji, Kyoto 611-0011, Japan. ${ }^{2}$ PRESTO, Japan Science and Technology Agency, 4-1-8 Honcho Kawaguchi, Saitama 332-0012, Japan. ${ }^{3}$ Graduate School of Science, Nagoya University, Chikusa, Nagoya 464-8602, Japan. Correspondence and requests for materials should be addressed to Y.M. (email: miyauchi@iae.kyoto-u.ac.jp).
} 
$\mathrm{P}$ hotoluminescence phenomena normally obey Stokes' law of luminescence $^{1}$, which states that the emission light wavelength (energy) is typically longer (lower) than its excitation counterpart. Up-conversion (or anti-Stokes) photoluminescence ${ }^{2}$ violates the Stokes' law of luminescence owing to additional energy gain induced by multiple photon ${ }^{2-5}$ or thermal (phonon) energy ${ }^{6,7}$ absorption. This unique photon up-conversion phenomenon only occurs efficiently in certain materials such as rare-earth-doped materials ${ }^{2,6,8}$ and some organic dyes ${ }^{3,4}$ by virtue of their specific electronic structures. Its potential usefulness in various applications such as photovoltaic energy conversion ${ }^{3,9}$, photoluminescence bioimaging $^{8,10-13}$, displays ${ }^{14}$, lasers ${ }^{15}$ and optical refrigeration of solids ${ }^{6,16}$ has attracted growing interest in recent years. However, especially in the near-infrared optical range, limitations in materials and combinations of excitation and emission photon energies available for efficient photon up-conversion have reduced its compatibility with a wide range of applications.

Single-walled carbon nanotubes (hereafter referred to as carbon nanotubes) consist of graphene sheets rolled up into quasi-one-dimensional tubular structures with diameters of the order of $1 \mathrm{~nm}$ (ref. 17). Depending on the rolling-up direction and their diameter defined by two indices $(n, m)^{18}$, nanotubes act as direct gap semiconductors with band gaps of the order of $1 \mathrm{eV}$ and emit photoluminescence in the near-infrared optical range ${ }^{19}$ because of recombination of excitons (electron and hole pairs bound via attractive Coulomb interactions) ${ }^{20-22}$. Since the first observation of efficient Stokes photoluminescence from individual carbon nanotubes ${ }^{19,23}$, numerous distinctive luminescence properties of nanotubes and their applications have been uncovered over the past decade ${ }^{24-27}$. However, this report reveals an additional remarkable characteristic of carbon nanotubes as photoluminescent materials.

Here, we demonstrate that carbon nanotubes ${ }^{17}$ are new upconversion phosphors showing efficient near-infrared to nearinfrared up-conversion photoluminescence at room temperature with an energy gain of up to a few hundreds of milli-electronvolts, corresponding to light wavelength shortening of a few hundreds of nanometres. This photon up-conversion does not originate from common coherent two-photon absorption or anti-Stokes Raman processes. The observed phenomena are attributed to efficient excitonic up-conversion processes gaining energy by absorbing high-energy $(\sim 100-200 \mathrm{meV})$ optical phonons characteristic in carbon nanotubes, although the phonon energy much exceeds the ambient thermal energy (only $\sim 26 \mathrm{meV}$ ) and corresponding phonon number available for the up-conversion is considerably small. This apparently anomalous process stems from unique excited-state dynamics emerging in an individual carbon nanotube with accidentally or intentionally embedded localized states. These findings may lead to new opportunities for near-infrared and thermal energy harvesting, optoelectronics and deep-tissue up-conversion photoluminescence imaging using conventional silicon-based image sensors and low-cost nearinfrared light sources.

\section{Results}

Optical imaging of the up-conversion light emission. Figure 1a shows an optical image of photoemission from $(6,5)$-rich carbon nanotubes dispersed in $\mathrm{D}_{2} \mathrm{O}$ (Methods section) in a standard quartz cell. The incident photon wavelength (energy) was set to ca. $1,100 \mathrm{~nm}(1.13 \mathrm{eV})$ and the photoemission image ranging between 950 and $1,000 \mathrm{~nm}(1.24-1.31 \mathrm{eV})$ was selectively captured using optical filters. The power density of the collimated incident light equalled $200 \mathrm{~mW} \mathrm{~cm}^{-2}$, which was too weak to induce coherent two-photon excitation photoluminescence ${ }^{21,22}$.
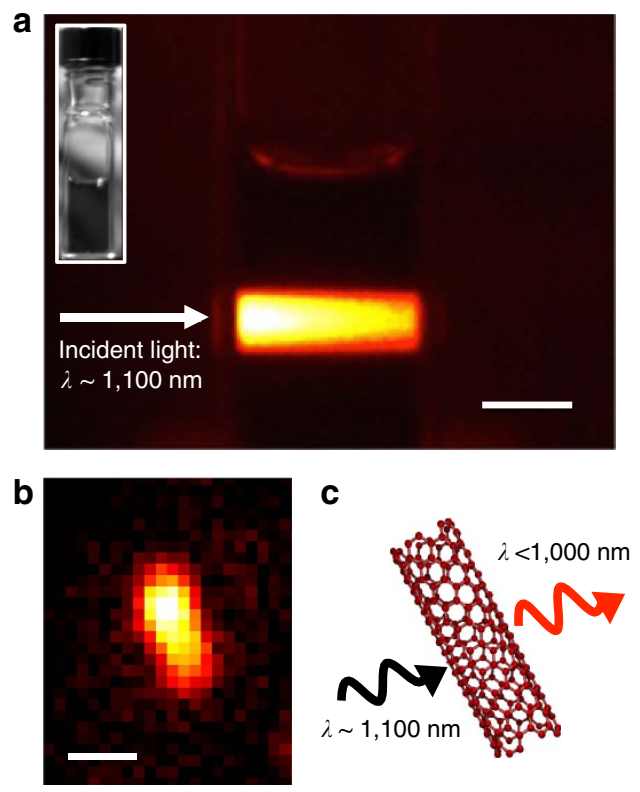

Figure 1 | Up-conversion light emission of carbon nanotubes. (a) Optical image showing light emission from $(6,5)$-rich carbon nanotubes dispersed in $\mathrm{D}_{2} \mathrm{O}$ using surfactant (sodium dodecyl benzene sulfonate, Methods section) excited by incident light of wavelength $\sim 1,100 \mathrm{~nm}$ (selected wavelength range of 1,075-1,125 nm, power density $=200 \mathrm{~mW} \mathrm{~cm}^{-2}$, collimated). The photoemission image was selectively detected in the 950-1,000 $\mathrm{nm}$ wavelength range using optical filters. The micellesuspended nanotube dispersion was placed in a quartz cell (inset). Scale bar, $5 \mathrm{~mm}$. (b) Microscopic image of the up-conversion light emission from an individual nanotube taken under the same excitation wavelength $(\sim 1,100 \mathrm{~nm})$ used in the experiment shown in a. Approximate excitation power density was $4 \mathrm{~kW} \mathrm{~cm}^{-2}$ (Methods section). Scale bar, $1 \mu \mathrm{m}$. (c) Optical processes observed in the experiment shown in a and $\mathbf{b}$. A shorter wavelength (higher energy) photoemission $(<1,000 \mathrm{~nm}$ ) results from excitation at longer wavelength (lower energy) incident light $(\sim 1,100 \mathrm{~nm})$.

Although the incident photon energy (wavelength) was considerably lower (longer) than the detected photon energy (wavelength), photoemission was clearly observed along the incident light path. We also confirmed that this light emission is from individual carbon nanotubes by directly observing microscopic light emission image of an individual nanotube under the up-conversion excitation condition as shown in Fig. 1b. This situation is schematized in Fig. 1c.

Up-conversion photoemission spectra. Figure 2a compares photoemission spectra of nanotubes excited with $1.13(1,100 \mathrm{~nm}$; red curve) and $2.18 \mathrm{eV}$ (568 nm; black curve) photons, respectively. The grey curve shows control data obtained for a nanotube-free surfactant solution excited at $1.13 \mathrm{eV}$. The emission peak detected at ca. $1.26 \mathrm{eV}$ upon excitation at $2.18 \mathrm{eV}$ (resonant with the second sub-band exciton energy $E_{22}$ of $(6,5)$ nanotubes) corresponds to the photoluminescence induced by exciton recombination in the first sub-band $\left(E_{11}\right)$ of $(6,5)$ nanotubes $^{23}$. The photoemission spectrum obtained at an excitation energy of $1.13 \mathrm{eV}$ exhibited a peak at ca. $1.26 \mathrm{eV}$. The similar peak energies for excitation energies of 1.13 and $2.18 \mathrm{eV}$ indicate that the photoemission mainly originates from $(6,5)$ nanotubes (Supplementary Note 1 and Supplementary Fig. 1).

Excitation power dependence. The excitation power density dependence was examined to clarify the origin of the observed 


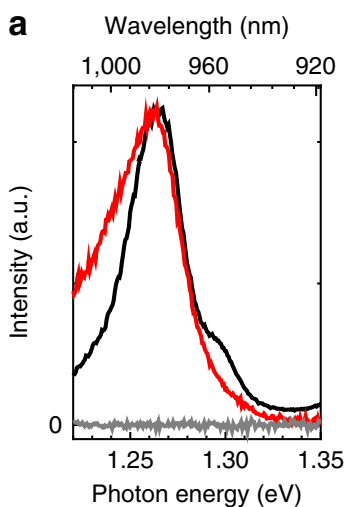

b

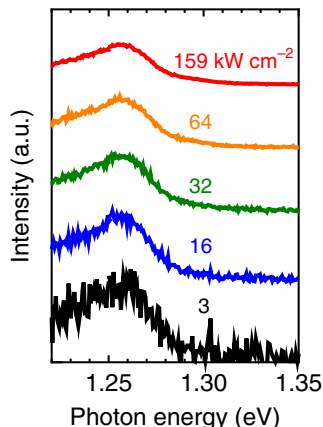

C

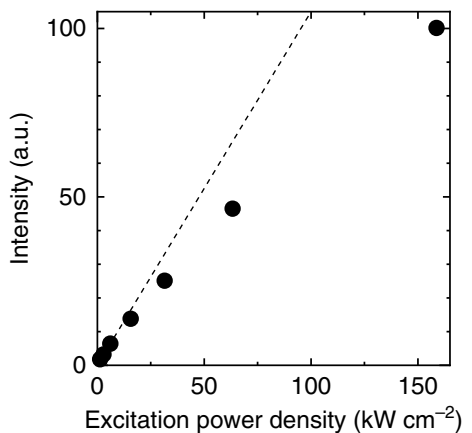

$f$
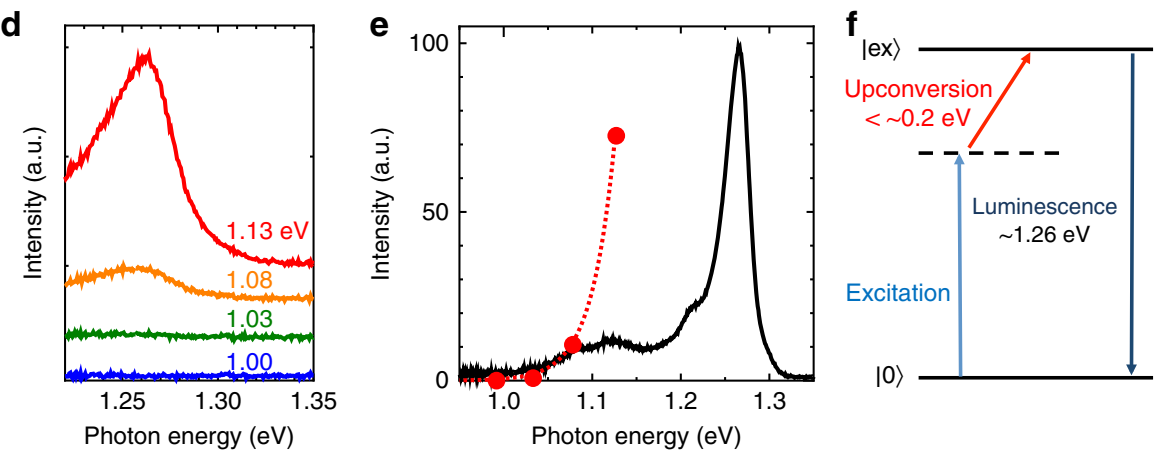

Figure 2 | Excitation power and energy dependences of the up-conversion photoemission. (a) Photoemission spectra of carbon nanotubes excited at incident photon energies of $1.13(1,100 \mathrm{~nm}$, red curve) and $2.18 \mathrm{eV}$ ( $568 \mathrm{~nm}$, black curve). The gray curve is a spectrum taken for a carbon nanotube-free surfactant solution excited at $1.13 \mathrm{eV}$ for comparison. The intensity was normalized so that the noise level appears to be comparable to the spectrum of carbon nanotubes excited at $1.13 \mathrm{eV}$ (red curve). The excitation band width was $10 \mathrm{~nm}$. (b) Excitation power density dependence of photoemission spectra excited at $1.13 \mathrm{eV}$. The spectra for excitation densities from 3 to $159 \mathrm{~kW} \mathrm{~cm}^{-2}$ are shown. Each spectrum is normalized by corresponding excitation power density. (c) Photoemission intensity (from data shown in b) plotted as a function of excitation power density. The dotted line corresponds to linear function for comparison. (d) Excitation photon energy dependence of the photoemission spectra. In $\mathbf{b}$ and $\mathbf{d}$, each spectrum was vertically shifted for comparison, and corresponding excitation power densities (for $\mathbf{d}$ ) or excitation photon energies (for d) are indicated on each spectrum for clarity. (e) Up-conversion photoemission intensity (red circles, from spectra in d) as a function of excitation photon energy, plotted together with normal photoluminescence spectrum at an excitation energy of $2.18 \mathrm{eV}$ (black curve) for comparison. The dotted red curve is a guide to the eye. (f) Energy diagram for the observed photon up-conversion processes. Solid lines represent the ground $(|0\rangle)$ and free exciton $(|e x\rangle)$ states in carbon nanotubes. The dashed line corresponds to an intermediate state in the up-conversion process.

up-conversion photoemission. The spectral shape hardly changed with excitation power density variations (Fig. 2b). Figure $2 c$ shows the up-conversion photoemission intensity as a function of excitation power density, revealing a weak sublinear dependence between these parameters. These observations exclude any possible photoemission induced by multiple-photon absorption (coherent or incoherent) or Auger recombination-mediated upconversion $^{28}$ as origins for the observed phenomena because these mechanisms usually display a superlinear (quadratic or more) excitation power dependence.

Excitation photon energy dependence. Next, the excitation photon energy dependence of the up-conversion photoemission spectra was evaluated (Fig. 2d). The up-conversion intensity strongly depended on the excitation photon energy (wavelength) but spectral shape and peak positions remain nearly intact upon excitation photon energy variations. This observation rules out the possibility that the up-conversion photoemission results from anti-Stokes Raman scattering because Raman processes induce clear peak shifts according to excitation energy changes.

The exclusion of these potential processes suggests that the observed up-converted photoemission is attributable to photoluminescence induced by one-photon absorption followed by an internal excitonic up-conversion process in a carbon nanotube.
Therefore, the observed phenomena are referred to as up-conversion photoluminescence in the remainder of the manuscript. Let us now consider the mechanism that governs this efficient excitonic up-conversion. The excitation photon energy dependence of the up-conversion intensity may provide a clue to the mechanism. Figure 2e compares the observed upconversion photoluminescence intensity (red circles) as a function of excitation photon energy with a normal (Stokes) photoluminescence spectrum (black curve) obtained at an excitation energy of $2.18 \mathrm{eV}$. The threshold excitation photon energy required to achieve an observable up-conversion photoluminescence reaching ca. $1.3 \mathrm{eV}$ amounted to $1.03-1.08 \mathrm{eV}$. A diagram showing excitation and emission photon energies for the observed up-conversion processes is shown in Fig. $2 \mathrm{f}$.

Temperature dependence. The threshold energy gain close to the maximum optical phonon energy $(\sim 0.2 \mathrm{eV})$ in carbon nanotubes ${ }^{29}$ and increasing up-conversion efficiency for smaller energy gain imply that phonon-assisted processes may induce internal upconversion in carbon nanotubes. Therefore, temperaturedependent studies on the up-conversion photoluminescence were conducted. Figure 3a compares up-conversion photoluminescence intensities at 297 and $281 \mathrm{~K}$ at an excitation energy of $1.13 \mathrm{eV}$. The up-conversion photoluminescence intensity exhibited high 

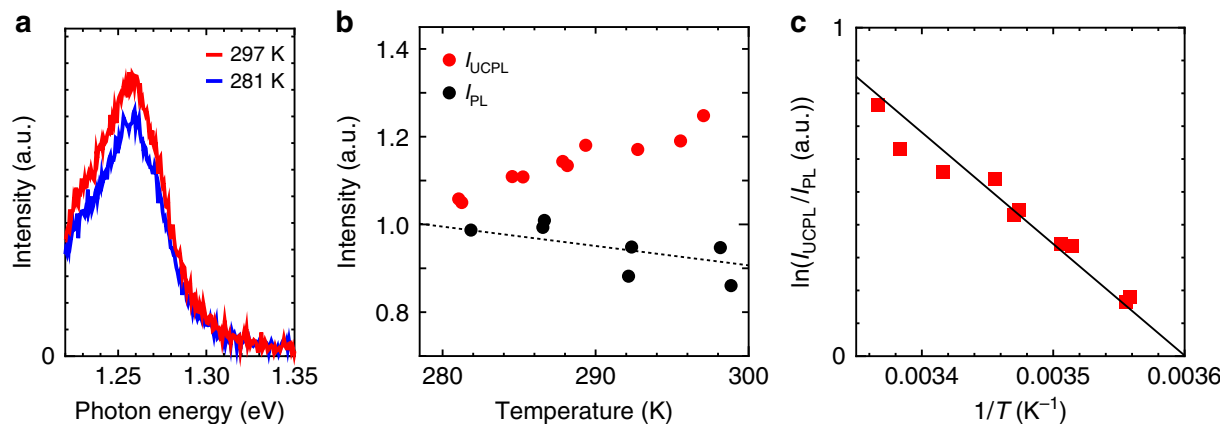

Figure 3 | Temperature dependence of the up-conversion photoluminescence. (a) Up-conversion photoluminescence spectra at 297 (red curve) and $281 \mathrm{~K}$ (blue curve) for an excitation energy of $1.13 \mathrm{eV}$. (b) Up-conversion (red circles, excitation energy $=1.13 \mathrm{eV}$, IUcPL) and normal photoluminescence intensities (black circles, excitation energy $=2.18 \mathrm{eV}, I_{\mathrm{PL}}$ ). The temperature-dependent normal photoluminescence intensity is fitted with a linear function (dotted line), which was used to normalize the up-conversion photoluminescence intensity at each temperature in c. (c) Arrhenius plot of the upconversion intensity ( $\left.I_{\mathrm{UCPL}}\right)$ normalized by the normal photoluminescence intensity $\left(I_{\mathrm{PL}}\right)$ at the corresponding temperature (red squares). $I_{\mathrm{UCPL}}$ was divided by $I_{P L}$ to account for the temperature-dependent variation of $I_{P L}$. The black solid line represents the Arrhenius equation for an activation energy of 120 meV.

sensitivity to small temperature variations and increased with increasing temperature. Figure $3 \mathrm{~b}$ shows the up-conversion (red open circles) and normal photoluminescence intensities (black open circles) as functions of temperature. Up-conversion and normal photoluminescence intensities displayed opposite trends with respect to temperature (Fig. 3b). The up-conversion photoluminescence intensity increased, whereas its normal counterpart slightly decreased when the temperature increased.

Figure $3 \mathrm{c}$ shows an Arrhenius plot of the up-conversion intensity $\left(I_{\mathrm{UCPL}}\right)$ divided by the normal photoluminescence intensity $\left(I_{\mathrm{PL}}\right)$ at each corresponding temperature. The solid line in Fig. $3 c$ represents the Arrhenius equation $\left(\propto \exp \left(-E_{\mathrm{a}} / k_{\mathrm{B}} T\right)\right.$ where $E_{\mathrm{a}}, k_{\mathrm{B}}$ and $T$ are the activation energy, Boltzmann constant and temperature) for an $E_{\mathrm{a}}$ value of $120 \mathrm{meV}$. (See also Supplementary Fig. 2 for data obtained at an excitation energy of $1.08 \mathrm{eV})$. The experimental trend was well reproduced with an $E_{\mathrm{a}}$ value closely matching the typical energy gain $(120-130 \mathrm{meV})$ at an excitation energy of $1.13 \mathrm{eV}$, strongly suggesting that the internal exciton up-conversion originates from phonon absorption processes in carbon nanotubes, rather than other possible processes such as triplet-triplet annihilation ${ }^{3,30}$. Note that the activation energy $E_{\mathrm{a}}$ nearly equal to the energy gain indicates onestep up-conversion via absorption of an optical phonon with much higher energy $(\sim 100-200 \mathrm{meV})$ than $k_{\mathrm{B}} T(\sim 26 \mathrm{meV})$, distinct from common thermal activation process in which the additional energy is mainly provided by absorption of multiple phonons with energy comparable to or lower than $k_{\mathrm{B}} T$.

Intermediate states in up-conversion processes. Now that the up-conversion photoluminescence has been clearly attributed to phonon-assisted processes, the reason for the efficient phononassisted up-conversion with an energy gain reaching 5-10 $k_{\mathrm{B}} T$ needs to be elucidated. The weak sublinear dependence of the upconversion photoluminescence intensity on the excitation power density (Fig. 2c) may provide insight into this anomalous phenomenon. The sublinear dependence implies the existence of an intermediate state with finite lifetime causing state-filling-induced saturation effect. Because it lies at lower energy than the $E_{11}$ free exciton state, this state may be a mid-gap localized state st-34 $^{31}$ unintentionally generated in carbon nanotubes during sample preparation because some types of these states ${ }^{32-36}$ are optically active and can be photoexcited directly by near-infrared photons.

To test this hypothesis, localized states were intentionally embedded in carbon nanotubes by chemical treatments ${ }^{32-34}$, and up-conversion photoluminescence changes were examined.
Carbon nanotubes with oxygen- and $\mathrm{sp}^{3}$-defect-derived optically active localized (zero-dimension (0D)-like) states were prepared according to procedures originally developed by Ghosh et al. $^{32}$ and Piao et al. ${ }^{34}$ with some modifications in selections of chemicals and experimental parameters, respectively (Methods section). Figure $4 \mathrm{a}, \mathrm{f}$ compare the normal photoluminescence spectra of as-dispersed (black curves) and $\mathrm{sp}^{3}$-defect (red curve) or oxygen-doped (blue curve; hereafter referred to as 0D-doped) carbon nanotubes. A relatively broad and intense photoluminescence band emerged around $1.1 \mathrm{eV}$ after chemical treatment, whereas the original $E_{11}$ photoluminescence intensities decreased only slightly for $0 D$-doped nanotubes. Figure $4 b-e, g-j$ show upconversion photoluminescence spectra of as-dispersed (black curves) and 0D-doped carbon nanotubes (coloured curves) excited at $1.13,1.08,1.03$ and $1.00 \mathrm{eV}$, respectively. Note that the 0D-doped nanotubes displayed a considerably enhanced up-conversion photoluminescence intensity compared with their as-dispersed counterparts regardless of origins of the localized states.

Figure 4k,l compare the excitation energy-dependent upconversion photoluminescence intensities of as-dispersed and 0D-doped nanotubes. The up-conversion photoluminescence intensity increased dramatically at the excitation energy of $1.08 \mathrm{eV}$ for 0D-doped nanotubes. Because this energy closely matched the photoluminescence peak energy of the localized excitons (Fig. 4a,f), this enhancement is attributable to exciton up-conversion from the additional localized states. The temperature (Supplementary Fig. 3) and the excitation power density dependences (Supplementary Note 2 and Supplementary Fig. 4) of the up-conversion photoluminescence were also assessed for the 0D-doped nanotubes. The results were similar to those obtained for as-dispersed nanotubes. This strongly suggests that the embedded localized states act as intermediate states in up-conversion processes. Moreover, the strong enhancement of the up-conversion photoluminescence achieved by simple chemical treatments is attractive for their applications as upconversion phosphors.

\section{Discussion}

We deduced the up-conversion quantum yield of the localized excitons to the free excitons using the difference between upconversion photoluminescence intensities before and after intentional localized state doping (see Supplementary Discussion and Supplementary Fig. 9 for details). It was of the order of $10^{-1}$ for an energy gain of $120 \mathrm{meV}$ even at room 
a
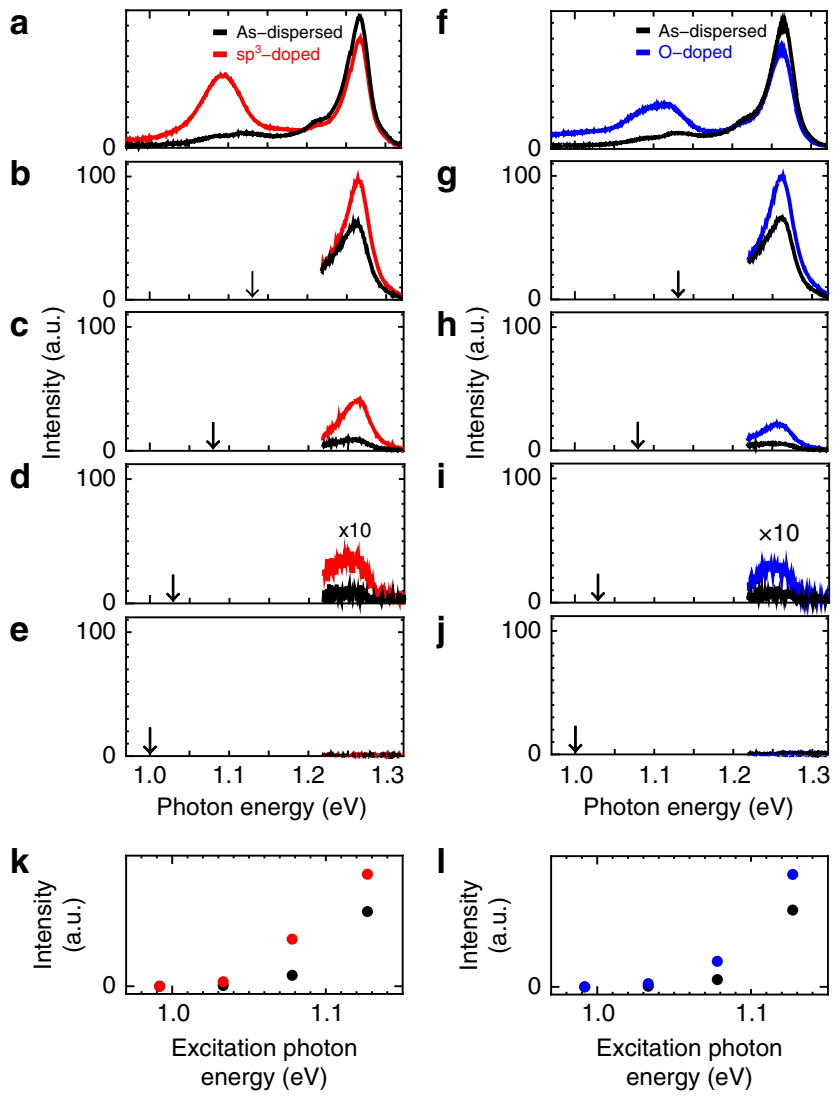

Figure 4 | Up-conversion photoluminescence with intentionally doped

states. (a,f) Photoluminescence spectra of as-dispersed (black curves) and OD-doped carbon nanotubes (red and blue curves for (a) $\mathrm{sp}^{3}$-and (f) oxygen-doped structures, respectively). $\mathbf{a}$ and $\mathbf{f}$ show normal photoluminescence spectra of each sample excited at $2.18 \mathrm{eV}$. (b-e, $\mathbf{g}-\mathbf{j})$ Up-conversion photoluminescence spectra of samples excited at $(\mathbf{b}, \mathbf{g}) 1.13$, $(\mathbf{c}, \mathbf{h}) 1.08,(\mathbf{d}, \mathbf{i}) 1.03$ and $(\mathbf{e}, \mathbf{j}) 1.00 \mathrm{eV}$, respectively. Vertical arrows in each panel indicate the corresponding excitation photon energy. Spectra in $\mathbf{d}, \mathbf{i}$ for an excitation energy of $1.03 \mathrm{eV}$ were magnified 10 times. (k,I) Excitation energy-dependent up-conversion photoluminescence intensities of asdispersed (black circles) and OD-doped (red and blue circles for $\mathrm{sp}^{3}$ - and oxygen-doped structures, respectively) carbon nanotubes.

temperature. This highly efficient phonon-assisted exciton upconversion showing an energy gain much exceeding the thermal energy appears anomalous but may be understood as a consequence of rapid exciton migration along the nanotube $\mathrm{axis}^{37}$ as described below. The efficient exciton scattering by acoustic phonons ${ }^{38}$ or random extrinsic surface potentials ${ }^{39}$ that drive rapid diffusive exciton migration ${ }^{37,40-44}$ surpassing $>100 \mathrm{~nm}$ along the nanotube axis spatially separates the upconverted excitons from their original localized state immediately after up-conversion and strongly reduces their probability of returning to this original state (see Supplementary Discussion and Supplementary Figs 10-12 for details). This unique exciton dynamics emerging in a carbon nanotube with embedded 0D-like localized states enables efficient phonon-assisted exciton up-conversion with an energy gain exceeding the thermal energy by one order of magnitude at room temperature. A potential enhancement of the exciton-phonon coupling resulting from exciton localization ${ }^{34,45,46}$ may also contribute to the efficient phonon-assisted exciton up-conversion in carbon nanotubes.

Finally, let us discuss the merits of the up-conversion excitation wavelength of the nanotubes lying in a specific near-infrared optical range $(\lambda=1,000-1,400 \mathrm{~nm})$. This wavelength range is known as 'second biological transparency window' (NIR II), in which the light penetration depth into biological tissues is maximized, whereas photo-induced degradation and autofluorescence are minimized ${ }^{25,47}$. Indeed, carbon nanotubes are expected to serve as promising phosphors for NIR II deeptissue imaging, and biocompatible and luminescent nanotube dispersion has already been developed ${ }^{25,47}$. A chief obstacle to this imaging approach has been the unsuitability of common silicon-based image sensors because of their vanishing sensitivity for NIR II photons. The up-conversion photoluminescence of the $(6,5)$ carbon nanotubes with the inverted excitation (in the NIR II range) and emission $(<1,000 \mathrm{~nm}$, so-called NIR I range) wavelengths may solve this problem and facilitate deep-tissue bio-imaging by taking advantage of the large penetration depth of NIR II photons and common silicon-based image sensors (Supplementary Notes 3-5 and Supplementary Figs 5-8). Because up-conversion occurs as a one-photon process in carbon nanotubes, it does not require costly ultrafast high power lasers and high-density excitation conditions unlike conventional multi-photon excitation imaging. Furthermore, up-converted photocarriers in carbon nanotubes may be directly extracted as electronic current by configuring photovoltaic devices because of their quasi-one-dimensional structures. Therefore, these findings may also lead to new routes for optoelectronics and heat and photon harvesting below the optical gap in the near-infrared region.

\section{Methods}

Sample preparation. (6,5)-rich CoMoCAT carbon nanotubes (Southwest NanoTechnologies, USA) were dispersed in $\mathrm{D}_{2} \mathrm{O}$ in the presence of $0.2 \%(\mathrm{w} / \mathrm{v})$ sodium dodecyl benzene sulfonate under moderate bath sonication for $60 \mathrm{~min}$ and vigorous sonication using a tip-type sonicator for $40 \mathrm{~min}$ before centrifugation at an acceleration of $130,000 \mathrm{~g}$ for $4 \mathrm{~h}$ (ref. 19). For the experiments shown in Fig. 4, additional localized states were embedded in carbon nanotubes by atomic oxygen ${ }^{32}$ or $\mathrm{sp}^{3}$-defect doping ${ }^{34}$ that reduce the band gap and exciton energy locally. Oxygen- and the $\mathrm{sp}^{3}$-defect-doped carbon nanotubes were prepared according to procedures originally developed by Ghosh et al. ${ }^{32}$ and Piao et al. ${ }^{34}$, respectively, with different chemical and experimental parameter selections ${ }^{33,48}$. In brief, the isolated nanotube dispersion was combined with a small amount of $\mathrm{D}_{2} \mathrm{O}$ containing dissolved ozone and left under the illumination of a desk lamp (ca. $5 \mathrm{~mW} \mathrm{~cm}^{-2}$ ) overnight, allowing moderate atomic oxygen doping to generate sparse oxygen-derived local states. To achieve $\mathrm{sp}^{3}$-defect doping, the nanotube dispersion was combined with a small amount of $\mathrm{D}_{2} \mathrm{O}$-containing dissolved organic 4-bromobenzenediazonium tetrafluoroborate salts ${ }^{34}$ and left in the dark for at least 10 days, allowing moderate $\mathrm{sp}^{3}$-defect doping in carbon nanotubes to generate sparse $\mathrm{sp}^{3}$-defect-derived localized electronic states. Optical absorption spectra of these samples are presented in Supplementary Fig. 1.

Optical measurements. Optical measurements were conducted using a broadband supercontinuum white light source (Fianium, SC450) for excitation. Incident light wavelengths were selected using a monochromator for normal Stokes emission measurements and optical filters for up-conversion photoemission measurements. The typical excitation band width was $10 \mathrm{~nm}$ for spectroscopic measurements and any unwanted stray light was carefully removed using multiple optical filters. Normal photoluminescence measurements were performed using monochromated light at a wavelength of $568 \mathrm{~nm}(2.18 \mathrm{eV})$ that matches the $E_{22}$ optical resonance in $(6,5)$ carbon nanotubes for photoexcitation. The optical image showing up-conversion photoemission phenomena (Fig. 1) was taken using an electron multiplying charge coupled device (EMCCD) camera (Princeton Instruments, ProEM). The microscopic up-conversion photoemission imaging of an individual nanotube shown in Fig. $1 \mathrm{~b}$ was performed using an inverted microscope and a 1.4 NA, $\times 100$ objective under epi-illumination of the near-infrared incident light $(1,075-1,125 \mathrm{~nm}$, approximate power density of $4 \mathrm{~kW} \mathrm{~cm}^{-2}$ ). For the microscopic imaging, the individually dispersed nanotubes were immobilized using an agarose gel matrix ${ }^{37}$. During spectral measurements shown in Figs 2-4, the nanotube dispersion was set in a quartz cell with optical path of $1 \mathrm{~mm}$. Incident light was focused on the sample in the quartz cell as a $4-6-\mu \mathrm{m}$ spot using an achromatic lens, and light emitted from the samples was collected by an achromatic lens and transferred to a confocal optical set-up. The near-infrared photoemission was recorded using a liquid nitrogen cooled Si-CCD or an InGaAs array detector. Detection sensitivity variations were corrected using a standard light source (Ocean Optics, LS-1). The spectral resolution typically equalled $2 \mathrm{~nm}$. We used spectral-integrated intensity for the analyses of the 
photoemission intensity as functions of excitation power density or temperature. The nanotube dispersion temperature was directly measured in a quartz cell using a dipped thin thermocouple. For the measurements of temperature dependence, nanotubes in the quartz cell were gradually cooled using iced water or allowed to warm up by natural convection. Optical spectra were acquired at temperatures ranging from $280 \mathrm{~K}$ to room temperature (ca. $300 \mathrm{~K}$ ). The temperature typically varied by $1.5 \mathrm{~K}$ during each spectral measurement.

\section{References}

1. Stokes, G. G. On the change of refrangibility of light. Philos. Trans. R. Soc Lond. 142, 463-562 (1852).

2. Auzel, F. Upconversion and anti-stokes processes with $\mathrm{f}$ and $\mathrm{d}$ ions in solids. Chem. Rev. 104, 139-174 (2004).

3. Baluschev, S. et al. Up-conversion fluorescence: noncoherent excitation by sunlight. Phys. Rev. Lett. 97, 143903 (2006).

4. Zhao, J., Ji, S. \& Guo, H. Triplet-triplet annihilation based upconversion: from triplet sensitizers and triplet acceptors to upconversion quantum yields. RSC Adv. 1, 937-950 (2011).

5. Joly, A. G., Chen, W., McCready, D. E., Malm, J.-O. \& Bovin, J.-O. Upconversion luminescence of cdte nanoparticles. Phys. Rev. B 71, 165304 (2005).

6. Sheik-Bahae, M. \& Epstein, R. I. Optical refrigeration. Nat. Photon. 1, 693-699 (2007).

7. Rakovich, Y. P. \& Donegan, J. F. in Semiconductor Nanocrystal Quantum Dots Ch. 9 (ed. Andrey, L. Rogach) 257-275 (Springer Vienna, 2008).

8. Wang, F. et al. Tuning upconversion through energy migration in core-shell nanoparticles. Nat. Mater. 10, 968-973 (2011).

9. Gray, V., Dzebo, D., Abrahamsson, M., Albinsson, B. \& Moth-Poulsen, K. Triplet-triplet annihilation photon-upconversion: towards solar energy applications. Phys. Chem. Chem. Phys. 16, 10345-10352 (2014).

10. Xu, C. T. et al. Autofluorescence insensitive imaging using upconverting nanocrystals in scattering media. Appl. Phys. Lett. 93, 171103 (2008).

11. Vinegoni, C. et al. Transillumination fluorescence imaging in mice using biocompatible upconverting nanoparticles. Opt. Lett. 34, 2566-2568 (2009).

12. Kotov, N. Bioimaging: The only way is up. Nat. Mater. 10, 903-904 (2011).

13. Dou, Q. Q., Guo, H. C. \& Ye, E. Near-infrared upconversion nanoparticles for bio-applications. Mater. Sci. Eng. C Mater. Biol. Appl. 45, 635-643 (2014).

14. Downing, E., Hesselink, L., Ralston, J. \& Macfarlane, R. A three-color, solidstate, three-dimensional display. Science 273, 1185-1189 (1996).

15. He, G. S., Markowicz, P. P., Lin, T.-C. \& Prasad, P. N. Observation of stimulated emission by direct three-photon excitation. Nature 415, 767-770 (2002).

16. Epstein, R. I., Buchwald, M. I., Edwards, B. C., Gosnell, T. R. \& Mungan, C. E. Observation of laser-induced fluorescent cooling of a solid. Nature 377, 500-503 (1995).

17. Iijima, S. \& Ichihashi, T. Single-shell carbon nanotubes of 1-nm diameter. Nature 363, 603-605 (1993).

18. Saito, R., Fuiita, M., Dresselhaus, G. \& Dresselhaus, M. S. Electronic structure of chiral graphene tubules. Appl. Phys. Lett. 60, 2204-2206 (1992).

19. O'Connell, M. J. et al. Band gap fluorescence from individual single-walled carbon nanotubes. Science 297, 593-596 (2002).

20. Ando, T. Excitons in carbon nanotubes. J. Phys. Soc. Jpn 66, 1066-1073 (1997).

21. Wang, F., Dukovic, G., Brus, L. E. \& Heinz, T. F. The optical resonances in carbon nanotubes arise from excitons. Science 308, 838-841 (2005).

22. Maultzsch, J. et al. Exciton binding energies in carbon nanotubes from twophoton photoluminescence. Phys. Rev. B 72, 241402 (R) (2005).

23. Bachilo, S. M. et al. Structure-assigned optical spectra of single-walled carbon nanotubes. Science 298, 2361-2366 (2002).

24. Avouris, P., Freitag, M. \& Perebeinos, V. Carbon-nanotube photonics and optoelectronics. Nat. Photon. 2, 341-350 (2008).

25. Welsher, K. et al. A route to brightly fluorescent carbon nanotubes for nearinfrared imaging in mice. Nat. Nanotechnol. 4, 773-780 (2009).

26. Miyauchi, Y. Photoluminescence studies on exciton photophysics in carbon nanotubes. J. Mater. Chem. C 1, 6499-6521 (2013).

27. Kruss, S. et al. Carbon nanotubes as optical biomedical sensors. Adv. Drug Deliv. Rev. 65, 1933-1950 (2013).

28. Ma, Y.-Z., Valkunas, L., Dexheimer, S. L., Bachilo, S. M. \& Fleming, G. R. Femtosecond spectroscopy of optical excitations in single-walled carbon nanotubes: evidence for exciton-exciton annihilation. Phys. Rev. Lett. 94, 157402 (2005)

29. Dresselhaus, M. S. \& Eklund, P. C. Phonons in carbon nanotubes. Adv. Phys. 49, 705-814 (2000).

30. Stich, D. et al. Triplet-triplet exciton dynamics in single-walled carbon nanotubes. Nat. Photon. 8, 139-144 (2014).

31. Crochet, J. J., Duque, J. G., Werner, J. H. \& Doorn, S. K. Photoluminescence imaging of electronic-impurity-induced exciton quenching in single-walled carbon nanotubes. Nat. Nanotechnol. 7, 126-132 (2012).

32. Ghosh, S., Bachilo, S. M., Simonette, R. A., Beckingham, K. M. \& Weisman, R. B. Oxygen doping modifies near-infrared band gaps in fluorescent single-walled carbon nanotubes. Science 330, 1656-1659 (2010).
33. Miyauchi, Y. et al. Brightening of excitons in carbon nanotubes on dimensionality modification. Nat. Photon. 7, 715-719 (2013).

34. Piao, Y. et al. Brightening of carbon nanotube photoluminescence through the incorporation of sp3 defects. Nat. Chem. 5, 840-845 (2013).

35. Ma, X. et al. Electronic structure and chemical nature of oxygen dopant states in carbon nanotubes. ACS Nano 8, 10782-10789 (2014).

36. Kilina, S., Ramirez, J. \& Tretiak, S. Brightening of the lowest exciton in carbon nanotubes via chemical functionalization. Nano Lett. 12, 2306-2312 (2012).

37. Cognet, L. et al. Stepwise quenching of exciton fluorescence in carbon nanotubes by single-molecule reactions. Science 316, 1465-1468 (2007).

38. Matsuda, K., Inoue, T., Murakami, Y., Maruyama, S. \& Kanemitsu, Y. Exciton dephasing and multiexciton recombinations in a single carbon nanotube. Phys. Rev. B 77, 033406 (2008).

39. Crochet, J. J. et al. Disorder limited exciton transport in colloidal single-wall carbon nanotubes. Nano Lett. 12, 5091-5096 (2012).

40. Rajan, A., Strano, M. S., Heller, D. A., Hertel, T. \& Schulten, K. Lengthdependent optical effects in single walled carbon nanotubes. J. Phys. Chem. B 112, 6211-6213 (2008).

41. Moritsubo, S. et al. Exciton diffusion in air-suspended single-walled carbon nanotubes. Phys. Rev. Lett. 104, 247402 (2010).

42. Hertel, T., Himmelein, S., Ackermann, T., Stich, D. \& Crochet, J. Diffusion limited photoluminescence quantum yields in 1-D semiconductors: single-wall carbon nanotubes. ACS Nano 4, 7161-7168 (2010).

43. Miyauchi, Y., Matsuda, K., Yamamoto, Y., Nakashima, N. \& Kanemitsu, Y. Length-dependent photoluminescence lifetimes in single-walled carbon nanotubes. J. Phys. Chem. C 114, 12905-12908 (2010).

44. Harrah, D. M. \& Swan, A. K. The role of length and defects on optical quantum efficiency and exciton decay dynamics in single-walled carbon nanotubes. ACS Nano 5, 647-655 (2011).

45. Perebeinos, V. \& Avouris, P. Phonon and electronic nonradiative decay mechanisms of excitons in carbon nanotubes. Phys. Rev. Lett. 101, 057401 (2008).

46. Galland, C., Högele, A., Türeci, H. E. \& Imamoğlu, A. Non-markovian decoherence of localized nanotube excitons by acoustic phonons. Phys. Rev Lett. 101, 067402 (2008).

47. Welsher, K., Sherlock, S. P. \& Dai, H. Deep-tissue anatomical imaging of mice using carbon nanotube fluorophores in the second near-infrared window. Proc Natl Acad. Sci. USA 108, 8943-8948 (2011).

48. Iwamura, M. et al. Nonlinear photoluminescence spectroscopy of carbon nanotubes with localized exciton states. ACS Nano 8, 11254-11260 (2014).

\section{Acknowledgements}

This research was supported by Precursory Research for Embryonic Science and Technology (PRESTO) from Japan Science and Technology Agency (JST)), Grants-in-Aid for Scientific Research (Nos. 24681031, 15H05408, 15K13337, 15K13500, 26107522 and 25400324) from Japan Society for the Promotion of Science (JSPS) and by Asahi Glass Foundation. We thank Y. Ogawa for experimental support, N. Nakashima for supplying raw materials and Y. Kawazoe, M. Uesugi, N. Tokitoh, T. Murakami, T. Umeyama and H. Imahori for experimental equipment.

\section{Author contributions}

Y.M. developed the concept, designed the experiment, and prepared the manuscript. N.A., S.A. and Y.M. performed the optical measurements and data analysis. N.A. prepared the samples. Y.M., N.A., S.M. and K.M. interpreted the results and wrote the manuscript. All authors discussed the results and commented on the manuscript.

\section{Additional information}

Supplementary Information accompanies this paper at http://www.nature.com/ naturecommunications

Competing financial interests: The authors declare no competing financial interests

Reprints and permission information is available online at http://npg.nature.com/ reprintsandpermissions/.

How to cite this article: Akizuki, N. et al. Efficient near-infrared up-conversion photoluminescence in carbon nanotubes. Nat. Commun. 6:8920 doi: 10.1038/ ncomms9920 (2015)

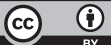

This work is licensed under a Creative Commons Attribution 4.0 International License. The images or other third party material in this article are included in the article's Creative Commons license, unless indicated otherwise in the credit line; if the material is not included under the Creative Commons license, users will need to obtain permission from the license holder to reproduce the material To view a copy of this license, visit http://creativecommons.org/licenses/by/4.0/ 